\section{Importancia del desarrollo de la motricidad fina en la etapa preescolar para la iniciación en la escritura}

\author{
Importance of the development of fine motor skills in the preschool stage for the \\ initiation in writing
}

Isabel Cristina Basto Herrera; Juan Carlos Barrón Parado; Luzmila Lourdes Garro-Aburto

\section{RESUMEN}

La escritura es una de las habilidades básicas para el desarrollo cognitivo del niño, es por eso que el artículo busca analizar la evidencia actual sobre la importancia del desarrollo de la motricidad fina en la etapa preescolar del infante para su iniciación en la escritura. Se realizó un proceso sistemático de revisión de artículos científicos indexados en base de datos relevantes como Scopus y otras, comprendidos en el periodo 2017 al 2020. Como resultado del estudio se presenta información vinculada a las categorías y subcategorías abordados en el trabajo. Se concluye que en el nivel inicial se enfatiza la adquisición de capacidades y aprendizajes fundamentales a través de actividades motrices, en este nivel el docente cumple un rol fundamental en el proceso del enfrentamiento del niño con la escritura. El nivel preescolar sienta las bases cognitivas y destrezas básicas en el niño para su acrecentamiento educativo y social.

Palabras clave: Escritura; desarrollo; motricidades finas; niños; preescolares

\section{ABSTRACT}

Writing is one of the basic skills for the cognitive development of the child, that is why the article seeks to analyze the current evidence on the importance of fine motor development in the preschool stage of the infant for its initiation in writing. A systematic process of review of scientific articles indexed in relevant databases such as Scopus and others, from 2017 to 2020, was carried out. As a result of the study, information linked to the categories and subcategories addressed in the work is presented. It is concluded that at the initial level, the acquisition of fundamental skills and learning is emphasized through motor activities; at this level, the teacher plays a fundamental role in the process of the child's confrontation with writing. The preschool level lays the cognitive foundations and basic skills for the child's educational and social development.

Keywords: Writing; development; fine motor skills; children; preschoolers
RELIGACIÓN

REVISTA DE CIENCIAS SOCIALES Y HUMANIDADES JOURNAL OF SOCIAL SCIENCES AND HUMANITIES REVISTA DE CENCIAS SOCIAIS E HUMANAS

INFORMACIÓN:

https://doi.org/10.46652/rgn.v6i30.834

ISSN 2477-9083

Vol. 6 No. 30, 2021. e210834

Quito, Ecuador

Enviado: agosto 30, 2021

Aceptado: octubre 25, 2021

Publicado: noviembre 19, 2021

Publicación Continua

Sección General | Peer Reviewed

\section{(c) $(\$)$}

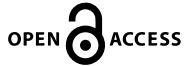

\section{AUTORES:}

Isabel Cristina Basto Herrera Universidad César Vallejo - Perú isabelbas@ucvvirtual.edu.pe

D Juan Carlos Barrón Parado Universidad César Vallejo - Perú jbarronpa@ucvvirtual.edu.pe

(D) Luzmila Lourdes Garro-Aburto Universidad César Vallejo - Perú lgarro@ucv.edu.pe

\section{CONFLICTO DE INTERESES}

Los autores declaran no tener conflicto de interés posible.

FINANCIAMIENTO

No existió asistencia financiera de partes externas al presente artículo. AGRADECIMIENTO

$\mathrm{N} / \mathrm{A}$

NOTA

El artículo no se desprende de un trabajo anterior.

ENTIDAD EDITORA 


\section{Introducción}

La escritura es una de las habilidades básicas para el desarrollo cognitivo del niño, por ello se debe estimular y cultivar a través de diferentes actividades motrices desde muy temprana edad, debido a que el saber y el aprender se centran en acciones gráfico-plástica como el dibujo, pintura, recorte, rasgado que el infante realiza por medio del ejercicio manual (Serrano \& De Luque, 2019; Uribe \& Torrealba, 2019), lo cual coadyuva a la mejora de la motricidad fina y se acrecienta con el juego libre (Dehghan et al., 2017; Escolano-Pérez et al., 2020). Entendemos por motricidad fina a los movimientos controlados y precisos que el infante realiza a través de la manipulación de objetos y materiales, lo cual tiene mayor desarrollo al ejecutar diversas tareas en la vida cotidiana y escolar (Syafril et al., 2018); el infante al tener dominio de sus movimientos incrementa su independencia manual como premisa para la iniciación de la escritura (Puente Balsells \& Viñals Carrera, 2019).

En el desarrollo de la motricidad fina la destreza gráfico plástica es crucial porque acrece la expresión, la comunicación socio afectivo y el aprendizaje motor en el infante (Vigo \& Torres, 2009). Según Piaget (1969) el niño adquiere el conocimiento a través de las acciones motrices (enfoque constructivista); para Lapierre \& Aucouturier (1983) el niño aprende a partir de su propio cuerpo, el objeto y el espacio sin dejar de lado lo afectivo y emocional (enfoque natural), y desde el enfoque global; el crecimiento, desarrollo, construcción de su esquema e imagen corporal del niño está vinculado entre lo mental y motriz, siendo la psicomotricidad el enlace de este binomio (Olano Rey, 1993). En este contexto, el nivel preescolar cumple un rol fundamental en la actuación motriz del niño (Alberto Díaz et al., 2017). Sin embargo, en este nivel se presta mayor atención a las competencias académicas, descuidando el progreso de la motricidad fina, provocando dificultad en el progreso adecuado de la habilidad motora fina, atención, concentración, coordinación viso-manual, manejo inadecuado de pinza para realizar diversos movimientos precisos en sus actividades diarias e incluso su predisposición para el siguiente nivel educativo.

En este contexto, el objetivo del artículo fue evidenciar la importancia del desarrollo de la motricidad fina en la etapa preescolar para su iniciación a la escritura.

\section{Metodología}

El artículo obedece a una revisión sistemática esta permitió analizar y evidenciar artículos y libros científicos vinculados al desarrollo de la motricidad fina en la etapa preescolar para la iniciación en la escritura. Se eligieron publicaciones de artículos indexados en base de datos como Scopus, Scielo, Redalyc, Dialnet, ProQuest, Latindex, publicados entre los años 2017 al 2020. Se realizó un proceso sistemático basado en las categorías y subcategorías abordados en el trabajo (Ver tabla 1). 
Tabla 1. Fuentes de revisión

\begin{tabular}{|c|c|c|}
\hline Categorías & Subcategorías & Autores \\
\hline $\begin{array}{l}\text { Habilidades } \\
\text { motoras finas }\end{array}$ & $\begin{array}{c}\text { Destreza manual } \\
\text { Habilidades manipulativas } \\
\text { Velocidad, fuerza y } \\
\text { precisión motora fina }\end{array}$ & $\begin{array}{c}\text { Qi, Y., Tan, S., Sui, M., \& Wang, J. (2018). } \\
\text { Dehghan, L., Mirzakhani, N., Rezaee, M., \& Tabatabaee, M. (2017). } \\
\text { Escolano-Pérez, E., Herrero-Nivela, M. L., \& Losada, J. L. (2020). } \\
\text { Gidion, H. (2020). } \\
\text { Syafril, S., Susanti, R., Fiah, R. El, Rahayu, T., Pahrudin, A., Yaumas, N. E., } \\
\text { \& Ishak, N. M. (2018). } \\
\text { McGlashan, H. L., Blanchard, C. C. V., Nicole, J. S., Lee, R., French, B., \& } \\
\text { Sycamore, N. J. (2017). } \\
\text { Taverna, L., Tremolada, M., Dozza, L., Zanin Scaratti, R., Ulrike, D., Lallo, } \\
\text { C., \& Tosetto, B. (2020). } \\
\text { Akin, S. (2019). } \\
\text { Barrera Erreyes, H. M., Flor Castelo, A. R., \& Flor Tapia, F. A. (2018). } \\
\text { Macías Merizalde, A. M., García Álvarez, I., Bernal Cerza, R. E., Zapata } \\
\text { Jaramillo, H. E., \& Álvarez García, I. (2020). }\end{array}$ \\
\hline $\begin{array}{l}\text { Práctica docente } \\
\text { para el desarrollo } \\
\text { de la motricidad } \\
\text { fina }\end{array}$ & $\begin{array}{l}\text { Preparación y } \\
\text { actualización }\end{array}$ & $\begin{array}{l}\text { Arteaga, I., Thornburg, K., Darolia, R., \& Hawks, J. (2019). } \\
\text { Andersson, S., Sandberg, G., \& Garpelin, A. (2019). } \\
\text { Cabrera Valdés, B. de la C., \& Dupeyrón García, M. de las N. (2019). }\end{array}$ \\
\hline $\begin{array}{c}\text { Coordinación } \\
\text { motora fina }\end{array}$ & $\begin{array}{l}\text { Desarrollo de tono muscu- } \\
\text { lar, lateralización, control } \\
\text { de movimientos y coor- } \\
\text { dinación de mano, brazo, } \\
\text { ojos y dedos }\end{array}$ & $\begin{array}{c}\text { Randjelović, N., Stanišić, I., Dragić, B., Piršl, D., \& Savić, Z. (2019). } \\
\text { Alvis, S. O., \& Llerena, A. J. P. (2020). }\end{array}$ \\
\hline $\begin{array}{l}\text { Empleo de } \\
\text { materiales }\end{array}$ & Diversidad de materiales & 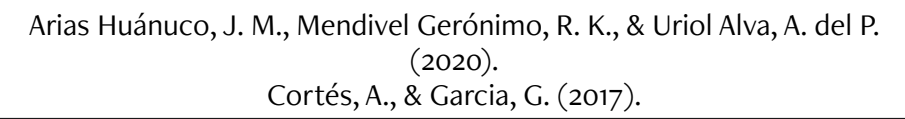 \\
\hline $\begin{array}{l}\text { Empleo de } \\
\text { ejercicios }\end{array}$ & $\begin{array}{l}\text { Preparación de ejercicios, } \\
\text { uso herramientas y mate- } \\
\text { riales }\end{array}$ & $\begin{array}{l}\text { Alberto Díaz, A., Bacallao Gallestey, J., Vargas-Machuca, R., Aguilar Ve- } \\
\text { larde, R., \& de citar, F. (2017). } \\
\text { Arias Huánuco, J. M., Mendivel Gerónimo, R. K., \& Uriol Alva, A. del P. } \\
\text { (2020). } \\
\text { Barrera Erreyes, H. M., Flor Castelo, A. R., \& Flor Tapia, F. A. (2018). } \\
\text { Cortés, A., \& Garcia, G. (2017). } \\
\text { Ojeda-Zamalloa, I., Serpa-Andrade, L., Quisi-Peralta, D., Vásquez-Álva- } \\
\text { rez, G., \& Quevedo-Sacoto, S. (2020). } \\
\text { Pérez Constante, M. B. (2017). } \\
\text { Portilla Castellanos, S. A., Rodríguez, M. N., \& Vera Silva, A. A. (2019). }\end{array}$ \\
\hline $\begin{array}{c}\text { Factores internos y } \\
\text { externos }\end{array}$ & $\begin{array}{l}\text { Familia, edad, género, } \\
\text { condiciones ambientales } \\
\text { y otros }\end{array}$ & $\begin{array}{c}\text { Cortés, A., \& Garcia, G. (2017). } \\
\text { Ramírez-Aguirre, G., Gutiérrez-Cedeño, M., León Piguave, A., Var- } \\
\text { gas-Cruz, M., \& Cetre-Vásquez, R. (2017). } \\
\text { Stone, R. (2019). }\end{array}$ \\
\hline
\end{tabular}

Fuente: Elaboración propia

\section{Desarrollo}

\subsection{Enfoques teóricos}

Piaget (1969) desde el enfoque constructivista refiere que las acciones motrices (psicomotricidad) repercuten en el acceso al conocimiento, en el desarrollo mental y en la conquista del mundo que rodea al niño a partir de las operaciones y los movimientos que realiza. Desde el enfoque global, el crecimiento y desarrollo del niño está vinculado entre el pensamiento (mental) y la acción (motriz), en donde la psicomotricidad es el enlace entre ellos, para su desarrollo y construcción de su esquema e imagen corporal (Olano Rey, 1993); y desde el enfoque natural, Lapierre \& Aucouturier (1983) reconocen que la motricidad se da a partir de su propio cuerpo, el objeto y el espacio, lo cual permite que el niño aprenda teniendo en cuenta el aspecto emocional y afectivo en ciertas situaciones fortificando su personalidad y el comportamiento. En efecto, el desarrollo mental está vinculado estrechamente con la motricidad del niño, ya que a través de ella expresa sus sentimientos, emociones, aprendizajes haciendo uso de la motricidad fina a través de sus producciones artísticas. 


\subsection{Definición motricidad fina}

Son movimientos controlados y precisos de los músculos de los ojos, lengua, manos y dedos al momento de utilizar herramientas y diversos objetos, tiene implicancia con el control de pequeños músculos del cuerpo que son los encargados del movimiento, lo cual conecta con las extremidades superiores en especial las manos que son la base de la capacidad del movimiento, implicando el funcionamiento de las destrezas, estructuras del cuerpo humano asociado a los hemisferios del cerebro (Motta \& Risueño, 2007; Serrano \& De Luque, 2019; Stassen Berger, 2007). La ausencia de la motricidad fina en los niños ocasiona torpeza motriz en la escritura, inseguridad, poco creativos, cansados, receptivos, memoristas, entro otros (Chuva-Castillo, 2016). La motricidad fina empieza a desarrollarse desde el primer momento de vida del niño y va en aumento según su aprendizaje y maduración, así como el incremento de la inteligencia de las habilidades gruesas y finas (Figura 1).

Figura 1. Categorías de la motricidad fina

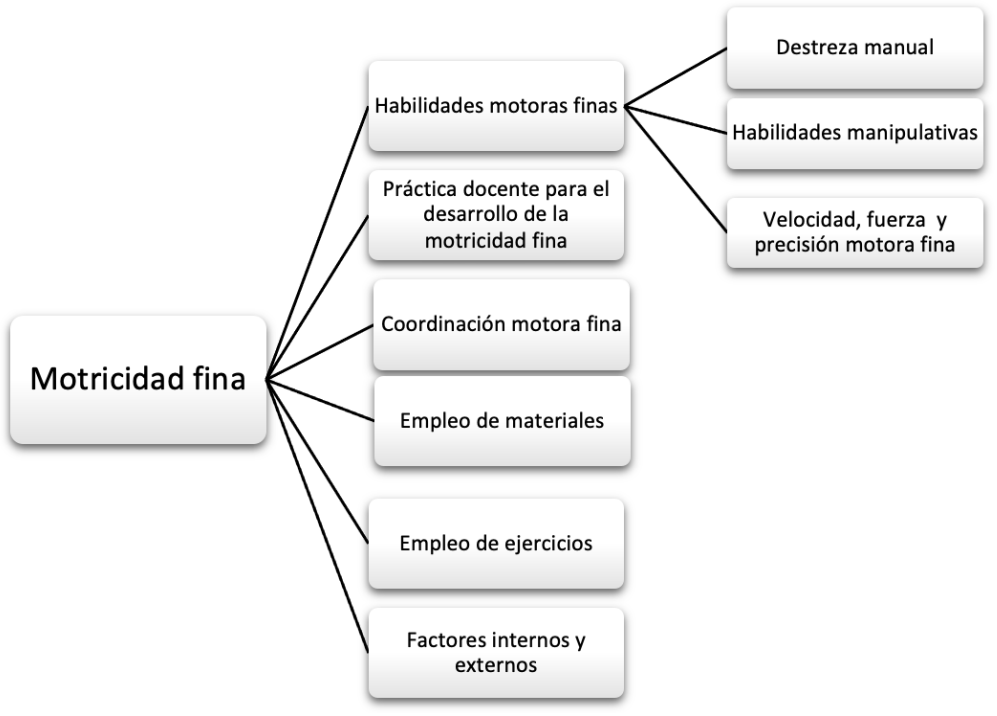

Fuente: Elaboración propia para de las categorías para el desarrollo de la motricidad fina.

\subsection{Habilidades motoras finas}

El desarrollo de las habilidades motoras finas conduce a realizar actividades de precisión, desarrollar habilidades sociales en los niños (madurez), habilidades de autoayuda e incluso mejorar su rendimiento académico; es adquirida a través de diferentes ejercicios, actividades y uso de diversos tipos de materiales que estimulan a los niños a ser entusiastas, estar felices y no darse por vencidos, favoreciendo significativamente a ser capaz de concentrarse, poseer buena fuerza en las manos y una excelente coordinación de ojos y manos (Qi et al., 2018). El desarrollo de estas habilidadesdebe iniciarse desde la primera infancia, ya que son flexibles durante esta etapa y se incrementa a través de las oportunidades basadas en el juego (Dehghan et al., 2017; Escolano-Pérez et al., 2020), teniendo encuenta la edad, conocimiento y crecimiento del niño para su capacidad de aprendizaje, además puede influenciar en el desarrollo de aspectos morales, espirituales, emocionales, de personalidad y su predisposición al ingresar al nivel primario (Gidion, 2020). El progreso de la motricidad fina en el niño preescolar se desarrollar a través de diversas acciones como el pintado con plastilina, juego origami, collage, pintado de dedos con temperas, juegos de Moronce, entre otros; los cuales ayudan a su destreza manual, habilidades manipulativas, velocidad, fuerza, precisión motora fina, coordinación manual y coordinación 
viso manual, lo que influirá en sus aprendizajes y disposición para ingresar al siguiente nivel. El empleo de ejercicios en la práctica y preparación para el perfeccionamiento de la motricidad fina por medio de actividades, propiciará bases para su posterior enfrentamiento a la escritura en el siguiente nivel escolar.

Para el perfeccionamiento y aumento gradual de estas habilidades proponen el uso del método de realizar collares utilizando cuentas, recortado, dibujo de formas básica y de personas (Macías Merizalde et al., 2020). Barrera Erreyes et al. (2018) propone punzado con palitos y articulación de marionetas. McGlashan et al. (2017) mecanografiar, entre otros. Estos métodos entrenan la coordinación e involucran los músculos de los dedos, ojos, manos y la madurez de los nervios para fortalecer la habilidad física y motora indispensables para el aprendizaje y progreso del infante sin dejar de lado su edad, perfil y su realidad.

\section{Destreza manual}

Es la capacidad de manipular los objetos con las manos, es esencial para los niños en la etapa prescolar, por que determina el rendimiento, velocidad y la legibilidad de la escritura a mano, se desarrolla a través de la práctica de ejercicios que implica equilibrio, coordinación motora fina y gruesa, la obtención de esta destreza influye en la autoestima y motivación en el niño, sin embargo la ausencia de esta afecta el rendimiento académico teniendo como consecuencia una escritura lenta y desigual (McGlashan et al., 2017). El desarrollo de esta destreza es fundamental para la instrucción y proceso de la escritura a mano, mejorando la articulación, aislamiento de los dedos, agarre en pinza, separación de los dedos pulgar e índice de la mano dominante de los niños para la escritura a mano de la etapa preescolar, incluso ayuda a la mejoría de la motricidad fina en los infantes con un déficit motor específico diagnosticado y con disgrafía (Taverna et al., 2020). En efecto, la destreza manual en los niños preescolar es es fundamental, ya que prepara al infante en el proceso de articulación para la escritura a mano y no tener más adelante una carencia motora específica.

\section{Habilidades manipulativas}

Son los movimientos que se centran en el manejo del objeto, desarrollada en forma progresiva a través de actividades lúdica para controlar se desarrolla en forma progresiva a través de variadas actividades lúdicas para controlar el movimiento y prensión palmar, radial, digital, tridigital y bidigital o trípode a través del sistema nerviso, músculos de los dedos y manos haciendo uso de materiales diversificados y atractivos que predispone de forma positiva a los niños (Barrera Erreyes et al., 2018; Macías Merizalde et al., 2020), lo que permitirá dar precisión a la mano, mejorando la legibilidad de la escritura, la escolarización y sus capacidades cognitivasdel niño (Akin, 2019). Definitivamente, las diferentes actividades manuales desarrolladas a través de actividades lúdicas y manipulación de diferentes materiales, son esenciales por que ayudan el normal desarrollo para la legibilidad de la escritura en los niños preescolar.

\section{Velocidad, fuerza y precisión motora fina}

Son factores indispensables para el desarrollo de habilidades en los sistemas sensoriomotores, el desarrollo de músculo grandes y pequeños, percepción visual, habilidades motoras finas, y habilidades en la mano que demuestran efecto positivo y significativo en el desarrollo de la articulación, instrucción, precisión y proceso de la escritura a mano en los niños preescolar ; esta se desarrolla a través de ejercicios y actividades contribuyendoa la fuerza prensil de manera efectiva y segura en los niños (Qi et al., 2018). En efecto, la velocidad, fuerza y precisión motora 
fina contribuyen a una articulación adecuada en el proceso de la escritura a mano de los niños preescolar es; así también, diversos ejercicios y actividades coadyuvan a sentirse seguro al momento de sujetar el lápiz, crayón u otro material.

\subsection{Práctica docente para el desarrollo de la motricidad fina}

El soporte que el maestro ofrece al niño preescolar es de gran relevancia para desafiar, estimular y guiar en su nacientes habilidades para una escritura creativa y funcional (Andersson et al., 2019). La preparación y actualización continua en métodos de enseñanza, creación de entornos eficaces para mejorar el aprendizaje y las habilidades de los niños en los maestros es fundamental por que conduce a un beneficio y progresonotable en la obtención de resultados positivos respecto a la ejecución de trazos con regularidad y precisión en los niños, igualmente el correcto uso de materiales, instrumentos y dominio de las técnicas (Arteaga et al., 2019; Cabrera Valdés \& Dupeyrón García, 2019). Definitivamente, la preparación continua del docente es primordial en el crecimiento de la motricidad fina en los niños preescolar es, porque son facilitadores y guías para la realización de actividades a través de diferentes materiales, correcto uso de instrumentos y dominio en el uso de las técnicas a desarrollar.

\section{Coordinación motora fina}

El entrenamiento de esta coordinación se realiza a través de diversas actividades y ejercicios que contribuye al progreso de la motricidad fina de los infantes para mejorar su coordinación y así desarrollar el tono muscular, lateralización, control voluntario de los movimientos y coordinación de la mano, brazo, ojos y los dedos, favoreciendo la preparación de una escritura en el nivel inicial con proyección al siguiente nivel, teniendo en cuenta los periodos de avance acorde a la edad del niño (Randjelović et al., 2019). Sabiendo que, a mayor desarrollo de la coordinación viso manual se favorecerá la capacidad de manipulación y el agarre en trípode, incrementando positivamente su coordinación motora fina en los infantes y por ende en el proceso manual en el inicio de la caligrafía en preescolar (Alvis \& Llerena, 2020). Indiscutiblemente, la coordinación motora fina en los niños preescolar es muy importante porque ayuda a la práctica satisfactoria de la caligrafía, lo cual le dará seguridad y tendrá mayor éxito, favoreciendo una escritura inicial y así continuar progresivamente al siguiente nivel.

\section{Empleo de materiales}

La utilización de diversos materiales es un elemento fundamental que provoca actividades y la construccion del conocimiento en el niño, tales como el papel craft, plastilina, témperas, colores, crayones, hojas de papel, lápices entre otros, es beneficioso e incrementa la motricidad fina en el niño preescolar (Arias Huánuco et al., 2020; Cortés \& Garcia, 2017). Indiscutiblemente el uso y variedad de materiales es muy significativo porque incrementa sus experiencias y ayuda a su desarrollo motriz fino de los niños.

\section{Empleo de ejercicios}

La práctica y preparación a través de adecuados ejercicios, actividades, uso de herramientas y materiales proporcionados en el periodo de práctica, estimula, acrecienta el desarrollo motriz y la creatividad en los niños, a través de movimientos de manipulación y digitales perfeccionandolas y preparando así las bases para su posterior enfrentamiento a la escritura (Pérez Constante, 2017; Portilla Castellanos et al., 2019). Al realizar diversos ejercicios como dibujo, coloreado 
de imágenes, tecnicas grafo plásticas fortalecen los músculos de los dedos, contribuyendo la realización y perfeccionamiento de trazos, imágenes, pulcritud, sincronización y exactitud, contribuyendo a un mejor aprestamiento (Barrera Erreyes et al., 2018; Cortés \& Garcia, 2017; Ojeda-Zamalloa et al., 2020; Portilla Castellanos et al., 2019). En las instituciones educativas de inicial se realizan diferentes tipos de ejercicios y actividades que favorecen la fuerza en los músculos pequeños, grandes y nervios tanto de las manos como de los dedos (Alberto Díaz et al., 2017; Arias Huánuco et al., 2020). Efectivamente, la práctica de ejercicios lleva mucho tiempo para la preparación, entrenamiento y concentración mental del niño, lo cual favorecerá en forma notable el incremento de la motricidad fina en los estudiantes preescolar es, lo que contribuirá a fortalecer las condiciones de su posterior enfrentamiento a la escritura

\subsection{Factores internos y externos}

La influencia de los factores internos como familia, edad, género, entre otros, y externos como condiciones ambientales, son determinantes y clave para el normal progreso del aprendizaje de la motricidad fina de los niños (Cortés \& Garcia, 2017; Stone, 2019). En los primeros cinco años del niño, la persona más cercana e influyente a él es generalmente la madre, considerada como un factor interno y clave trascendental en el progreso de su motricidad fina: desarrollo del sistema nervioso y habilidades físicas que hacen posible motivar el movimiento ya que el incremento en este periodo establecerá la independencia y preparación de los niños a la siguiente fase escolar (Ramírez-Aguirre et al., 2017; Stone, 2019). Sin duda, para el incremento de la motricidad fina en el infante se debe tomar en consideración los factores internos y externos siendo clave la persona más cercana para su progreso, sin dejar de lado las otras condiciones.

\section{Conclusiones}

El desarrollo de la motricidad fina en el niño preescolar, enfatiza la adquisición de diversas capacidades y aprendizajes fundamentales que se adquiere a través de diversas actividades y materiales que se utilizan. Este desarrollo debe darse en forma progresiva para ir perfeccionándolos, de manera que los estudiantes manifiesten su nivel de crecimiento a través de su sentir, pensamientos y experiencias, plasmadas en sus producciones artísticas optimizando su aprendizaje. Los resultados encontrados sobre la importancia de la motricidad fina asociado a la escritura en la etapa preescolar es prometedor, sugerimos el incremento de indagaciones sobre esta problemática.

El progreso de la motricidad fina en el niño preescolar se desarrolla a través de tareas escolares como el pintado con plastilina, juego origami, collage, pintado de dedos con temperas, juegos de Moronce, entre otros; los cuales ayudan a su destreza manual, habilidades manipulativas, velocidad, fuerza, precisión motora fina, coordinación manual y coordinación viso manual, lo que influirá en sus aprendizajes y disposición para ingresar al siguiente nivel. La aplicación constante de ejercicios en la práctica y preparación para el perfeccionamiento de la motricidad fina por medio de actividades, propicia bases para su posterior nivel educativo.

En el enfrentamiento del niño preescolar hacia la escritura el docente cumple un rol fundamental, ya que el empleo correcto de instrumentos, materiales y el dominio de las técnicas en la enseñanza hará ameno este proceso. La familia, edad, género y las condiciones ambientales son factores determinantes en el normal progreso y avance de la motricidad fina en los infantes. 


\section{Referencias}

Alkin, S. (2019). Fine Motor Skills, Writing Skills and Physical Education Based Assistive Intervention Program in Children at Grade 1. Journal Publishing Group Asian Journal of Education and Training, 5(4), 518525. https://doi.org/10.20448/journal.522.2019.54.518.525

Alberto Díaz, A., Bacallao Gallestey, J., Vargas-Machuca, R., Aguilar Velarde, R., \& de citar, F. (2017). Desarrollo infantil en zonas pobres de Perú Investigación original. Rev Panam Salud Publica, 41, 1-8. https:// dx.doi.org/10.26633\%2FRPSP.2017.71

Alvis, S. O., \& Llerena, A. J. P. (2020). Motricidad fina e Iniciación de la Caligrafía en niños de 5 años de Educación Inicial Yarinacocha, Perú. SENDAS, 1(1), 10. https://doi.org/10.47192/RCS.V111.21

Andersson, S., Sandberg, G., \& Garpelin, A. (2019). To teach writing: Teachers' perspectives on how to promote children's writing development in the Swedish preschool class. Nordic Journal of Literacy Research, 5(2), 23-38. https://doi.org/10.23865/njlr.v5.1460

Arias Huánuco, J. M., Mendivel Gerónimo, R. K., \& Uriol Alva, A. del P. (2020). La psicomotricidad en la preescritura de los niños de 5 años de las instituciones educativas de inicial del cercado de Huancavelica. Revista Conrado, 16(2020), 43-50. https://conrado.ucf.edu.cu/index.php/conrado/article/view/1451

Arteaga, I., Thornburg, K., Darolia, R., \& Hawks, J. (2019). Improving Teacher Practices With Children Under Five: Experimental Evidence From the Mississippi Buildings Blocks. Evaluation Review, 43(1-2), 41-76. https://doi.org/10.1177/0193841X19865070

Barrera Erreyes, H. M., Flor Castelo, A. R., \& Flor Tapia, F. A. (2018). Estimulación temprana y desarrollo psicomotor en niños de 4 a 5 años. Ciencia Digital, 2(1), 61-74. https://doi.org/10.33262/cienciadigital. v2i1.5

Cabrera Valdés, B. de la C., \& Dupeyrón García, M. de las N. (2019). El desarrollo de la motricidad fina en los niños y niñas del grado preescolar. Revista de Educación, 17(2), 1815-7696. http://mendive.upr.edu.cu/ index.php/MendiveUPR/article/view/1499

Chuva-Castillo, P. G. (2016). Desarrollo de la motricidad fina a través de técnica gráfico plásticas en niños de 3 á 4 años de la escuela de educación básica Federico Gonzáles Suarez [Tesis, Universidad Politécnica Salesiana]. Repositorio Institucional de la Universidad Politécnica Salesiana http://dspace. ups.edu.ec/handle/123456789/1273

Cortés, A., \& Garcia, G. (2017). Estrategias pedagógicas que favorecen el aprendizaje de niñas y niños de o a 6 años de edad en Villavicencio- Colombia. Revista Interamericana de Investigación Educación y Pedagogía RIIEP, 10(1), 125-143. https://doi.org/10.15332/S1657-107X.2017.0001.06

Dehghan, L., Mirzakhani, N., Rezaee, M., \& Tabatabaee, M. (2017). The relationship between fine motor skills and social development and maturation. Iranian Rehabilitation Journal, 15(4), 407-414. https:// doi.org/10.29252/nrip.irj.15.4.407

Escolano-Pérez, E., Herrero-Nivela, M. L., \& Losada, J. L. (2020). Association Between Preschoolers' Specific Fine (But Not Gross) Motor Skills and Later Academic Competencies: Educational Implications. Frontiers in Psychology, 11(1044), 1-19. https://doi.org/10.3389/fpsyg.2020.01044

Gidion, H. (2020). The Importance of measuring fine motor skill in early Children's Education. Atlantis Press, 426, 313-319. https://doi.org/10.2991/assehr.k.200331.160

Lapierre, A., \& Aucouturier, B. (1983). Simbología del movimiento: psicomotricidad y educación (2a.). Científico-Medica. https://cutt.ly/aEMt4XH

Macías Merizalde, A. M., García Álvarez, I., Bernal Cerza, R. E., Zapata Jaramillo, H. E., \& Álvarez García, I. (2020). La estimulación y el desarrollo motor fino en niños de 5 años. Revista pedagógica de la Universidad de Cienfuegos, 16(74), 306-311. https://conrado.ucf.edu.cu/index.php/conrado/article/view/1366

McGlashan, H. L., Blanchard, C. C. V., Nicole, J. S., Lee, R., French, B., \& Sycamore, N. J. (2017). Improvement in children's fine motor skills following a computerized typing intervention. Human Movement Science, 56, 29-36. https://doi.org/10.1016/j.humov.2017.10.013

Motta, I. M., \& Risueño, A. E. (2007). El juego en el aprendizaje de la escritura: fundamentación de las estrategias lúdicas. Editorial Bonum. 
Ojeda-Zamalloa, I., Serpa-Andrade, L., Quisi-Peralta, D., Vásquez-Álvarez, G., \& Quevedo-Sacoto, S. (2020). Diseño, construcción e implementación de una herramienta para el soporte para ejercicios de grafo-motricidad en niños de cuatro a seis años. Revista Ibérica de Sistemas e Tecnologias de Informação, E33(1), 263-274. https://doi.org/10.12738/ESTP.2019.1.0269

Olano Rey, R. (1993). La psicología genético-dialéctica de H. Wallon y sus implicaciones educativas (1ra.). Universidad de Oviedo. https://cutt.ly/8EoX1LX

Pérez Constante, M. B. (2017). Habilidades del área motriz fina y las actividades de estimulación temprana. Revista Publicando, 4(11(1)), 526-537. https://revistapublicando.org/revista/index.php/crv/article/ view/581

Piaget, J. (1969). Psicología y pedagogía. (2a.). Ariel. https://cutt.ly/7EXMDGM

Portilla Castellanos, S. A., Rodríguez, M. N., \& Vera Silva, A. A. (2019). La lectura y la escritura, una ventana al universo de los niños. Research report, 30, 63-81. https://www.proquest.com/docview/2260078391/ DCC510FB8C 384F6CPQ/2

Puente Balsells, M. L., \& Viñals Carrera, F. (2019). Grafología digital, tipográfica y del diseño visual (UOC (ed.); 1ra.). https://cutt.ly/HEXMBXk

Qi, Y., Tan, S., Sui, M., \& Wang, J. (2018). O treinamento físico supervisionado melhora as habilidades motoras finas em crianças de 5 anos. Revista Brasileira de Medicina do Esporte, 24(1), 9-12. https://doi. org/10.1590/1517-869220182401177117

Ramírez-Aguirre, G., Gutiérrez-Cedeño, M., León Piguave, A., Vargas-Cruz, M., \& Cetre-Vásquez, R. (2017). Coordinación grafoperceptiva: incidencia en el desarrollo de la motricidad fina en niños de 5 á 6 años de edad. Revista Ciencia Unemi, 10(22), 40-47. https://doi.org/10.29076/issn.2528-7737vol10iss22. 2017pp40-47p

Randjelović, N., Stanišić, I., Dragić, B., Piršl, D., \& Savić, Z. (2019). The sequence of procedures in the development of fine motor coordination through physical activities and movement games in preschool children. Facta Universitatis, Series: Physical Education and Sport, 16(3), 611-620. https://doi.org/10.22190/ fupes180607055r

Serrano, P., \& De Luque, C. (2019). Motricidad fina en niños y niñas: desarrollo, problemas, estrategias de mejora y evaluación. Narcea.

Stassen Berger, K. (2007). Psicología del Desarrollo. Infancia y adolescencia. Panamericana

Stone, R. (2019). Lectoescritura inicial en Latinoamérica y el Caribe: una revisión sistemática. Revista de Investigación y Evaluación Educativa, 6(1), 22-37. https://doi.org/10.47554/REVIE2019.6.28

Syafril, S., Susanti, R., Fiah, R. El, Rahayu, T., Pahrudin, A., Erlina, N., \& Ishak, N. M. (2018). Four Ways of Fine Motor Skills Development in Early Childhood. ResearchGate, 2018, 1-15. https://doi.org/10.31227/OSF. IO/PXFKQ

Taverna, L., Tremolada, M., Dozza, L., Zanin Scaratti, R., Ulrike, D., Lallo, C., \& Tosetto, B. (2020). Who Benefits from An Intervention Program on Foundational Skills for Handwriting Addressed to Kindergarten Children and First Graders? International Journal of Environmental Research and Public Health, 17(6), 1-24. https://doi.org/10.3390/ijerph17062166

Uribe, N. V., \& Torrealba, J. R. (2019). Ficción y realidad sociomotriz en la figura mítica del extraterrestre: desde la hipocinesia hacia la torpeza y pandemia. Revista Actividad Física y Ciencias Año, 11(1), 1-13. http://revistas.upel.digital/index.php/actividadfisicayciencias/article/view/7836

Vigo, C., \& Torres, S. (2009). Educación Inicial y Primaria en el Perú. Inversión en la infnancia. https://inversionenlainfancia.net/blog/entrada/entrevista/32/o

\section{AUTORES}

Isabel Cristina Basto Herrera. Magister en Psicopedagogía por la Universidad César Vallejo - Perú. Profesora de Educación Inicial.

Juan Carlos Barrón Parado. Magister en Docencia y Gestión Educativa por la Universidad Cesar Vallejo- Perú. Docente de educación básica en el nivel de educación primaria.

Luzmila Lourdes Garro-Aburto. Doctora en Psicología educacional y tutorial, Magíster en Tecnología educativa y Licenciada en Educación. Estudios de Posdoctorado en Estrategias e instrumentos de evaluación del desempeño en CIFE-México. Docente investigador a cargo de las asignaturas de Investigación de la Escuela de posgrado de la 